\title{
High Loading Capacity Nanoencapsulation and Release of Hydrophobic Drug Nanocrystals from Microgel Particles
}

\author{
Trystan Domenech, Patrick S. Doyle* \\ Department of Chemical Engineering, Massachusetts Institute of Technology, \\ Cambridge, Massachusetts 02139, USA
}

\section{Contents}

Supporting Methods and Data

Supporting Tables

Supporting Figures 


\section{Supporting Methods and Data}

Calculation of Inter-Nanocrystal Separation in the Dried Microgel Particles. The separation between the fenofibrate nanocrystals within the dried microgel particles was calculated based

on the nanocrystals volume fraction in the ried particles $\phi_{d}^{d r y}$. The latter can be expressed as follows (see the main text of the article for the definition of each variable):

$$
\phi_{d}^{d r y}=\frac{C_{f e n}}{\rho_{\text {cryst }}}\left(\frac{1}{S_{r}}\right)^{3} \phi_{d}
$$

Assuming evenly space spherical nanocrystals, one can deduce the inter-nanocrystal separation distance $\delta_{n c}$ as follows ${ }^{1}:$

$$
\delta_{n c}=\left(\left(\frac{\phi_{M}}{\phi_{d}^{d r y}}\right)^{3}-1\right) \cdot d_{c r y s t}
$$

where $\phi_{M}=0.64$ is the maximum packing volume fraction for monodisperse spheres, and $d_{\text {cryst }}$ is the nanocrystal diameter. The results are presented as a function of the particles' drug load in Figure S5.

Effect of Sieving Procedure on the Release Profile of Particles. Control experiments were performed in order to determine the influence of particles separation by sieving on the dissolution results. These experiments were conducted on dried $239 \mu \mathrm{m}$ particles with $70.7 \%$ drug load. Two particle preparation procedures were followed: in procedure 1, particles dried in contact with each other as a large batch were de-agglomerated via sieving through a $250 \mu \mathrm{m}$ mesh size; in procedure 2, a smaller batch of particles were dried far apart from each other in order to prevent particles agglomeration.

As can be seen in Figure S6, the release profiles obtained for both procedures are nearly identical, with almost no difference in $\mathrm{t}_{80}$ (time at $80 \%$ release). It is therefore concluded that the sieving procedure (denoted here as procedure 1), which allows for the drying and subsequent deagglomeration of large batches of particles, does not introduce any experimental artifact and was thus employed for this study.

\footnotetext{
${ }^{1}$ Hao, T.; Riman, R. E., Calculation of interparticle spacing in colloidal systems. J. Colloid Interface Sci. 2006, 297, (1), 374-377.
} 


\section{Supporting Tables}

Table S1. Specific enthalpy of fusion measured by differential scanning calorimetry and crystallinity of fenofibrate nanocrystals loaded in the particles. The specific enthalpy of fusion of bulk fenofibrate is $\Delta H_{\text {bulk }}=93.9 \mathrm{~J} / \mathrm{g}$. Crystallinity of the fenofibrate nanocrystals is defined as: $100 \% \cdot \Delta H_{\text {crystals }} / \Delta H_{\text {bulk }}$.

\begin{tabular}{cccc}
\hline Particle drug load $(\%)$ & $\Delta H_{\text {particles }}(\mathrm{J} / \mathrm{g})$ & $\Delta H_{\text {crystals }}(\mathrm{J} / \mathrm{g})$ & Crystallinity $(\%)$ \\
\hline 12.6 & $11.2 \pm 0.7$ & $89.2 \pm 5.7$ & $95.0 \pm 6.1$ \\
37.2 & $33.2 \pm 1.8$ & $89.3 \pm 4.9$ & $95.1 \pm 5.2$ \\
44.4 & $43.7 \pm 2.2$ & $98.6 \pm 5.0$ & $104.9 \pm 5.3$ \\
50.8 & $50.0 \pm 2.5$ & $98.3 \pm 4.9$ & $104.7 \pm 5.2$ \\
60.8 & $60.3 \pm 3.0$ & $99.2 \pm 4.9$ & $105.6 \pm 5.3$ \\
70.7 & $64.3 \pm 4.9$ & $91.0 \pm 4.9$ & $96.9 \pm 5.3$ \\
\hline
\end{tabular}

$\Delta H_{\text {bulk }}, \Delta H_{\text {particles }}$ and $\Delta H_{\text {crystals }}$ correspond to the specific enthalpies of fusion of bulk fenofibrate, particles and crystals loaded in the particles, respectively.

Table S2. Amounts of particles used for USP dissolution testing and fitting parameters of the Weibull function.

\begin{tabular}{ccccc}
\hline Particle drug load $(\%)$ & Particle size $(\mu \mathrm{m})$ & $M_{d}(\mathrm{mg})$ & $\tau_{d}(\mathrm{~min})$ & $\beta(-)$ \\
\hline 12.6 & 250 & 136.2 & 7.7 & 1.09 \\
37.2 & 250 & 45.9 & 7.6 & 0.98 \\
44.4 & 250 & 38.6 & 24.7 & 0.69 \\
50.8 & 250 & 33.7 & 67.4 & 0.70 \\
60.8 & 250 & 28.1 & 82.8 & 0.70 \\
70.7 & 250 & 24.2 & 93.5 & 0.73 \\
70.7 & 45 & 24.2 & 12.3 & 0.87 \\
70.7 & 90 & 24.2 & 28.1 & 0.76 \\
70.7 & 125 & 24.2 & 36.7 & 0.74 \\
\hline
\end{tabular}

$M_{d}$ is the mass of particles used for dissolution in $900 \mathrm{~mL}$ of dissolution medium, adjusted for each drug load in order to keep the fenofibrate concentration in the same sink conditions $(\mathrm{c} \approx 19 \mu \mathrm{g} / \mathrm{mL}) \cdot \tau_{d}$ and $\beta$ correspond to the characteristic dissolution time and shape parameter of the Weibull function, respectively. 


\section{Supporting Figures}

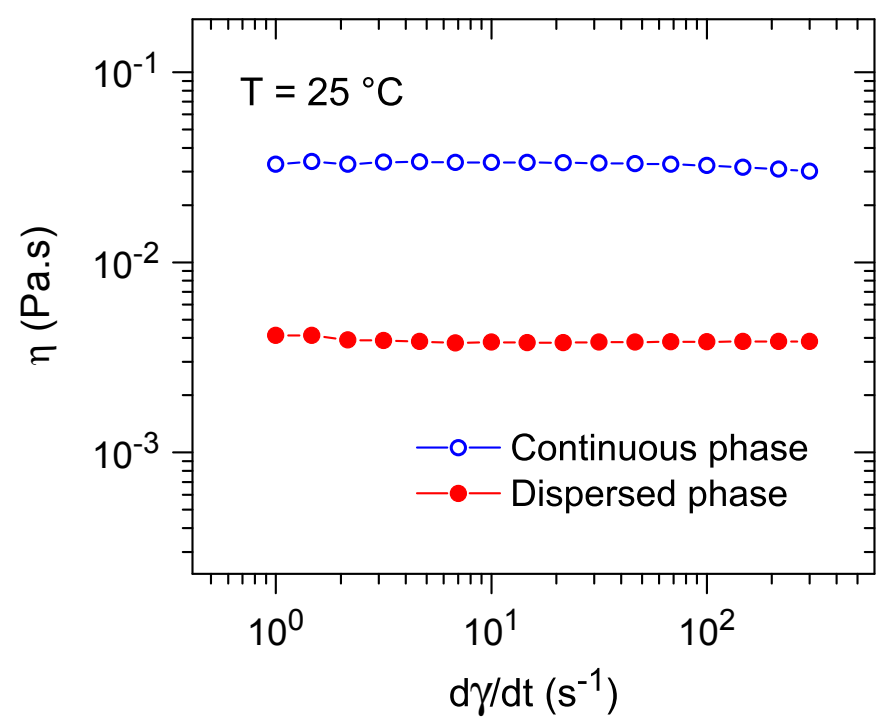

Figure S1. Flow curves (viscosity vs. shear rate) for the continuous phase (solution of 2 wt\% alginate in deionized water) and dispersed phase (saturated solution of fenofibrate in anisole).
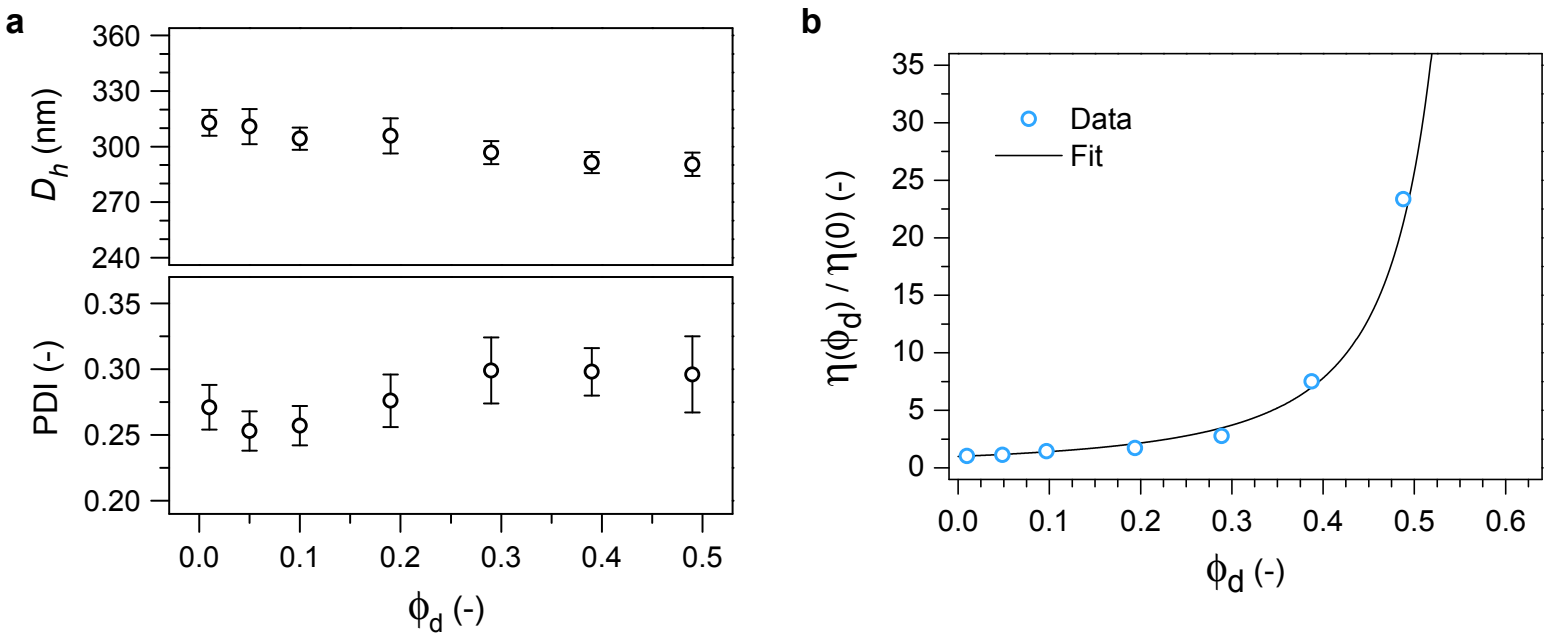

Figure S2. a Nanoemulsions hydrodynamic diameter $\left(D_{h}\right)$ and polydispersity index (PDI) as a function of the dispersed phase volume fraction $\left(\phi_{\mathrm{d}}\right)$, measured by dynamic light scattering. $\mathbf{b}$ Fitting of the nanoemulsion/continuous phase viscosity ratio $\left(\eta_{r}\right)$ as a function of the dispersed phase volume fraction using the Quemada equation. Viscosities were measured at a shear rate of $300 \mathrm{~s}^{-1}$. 

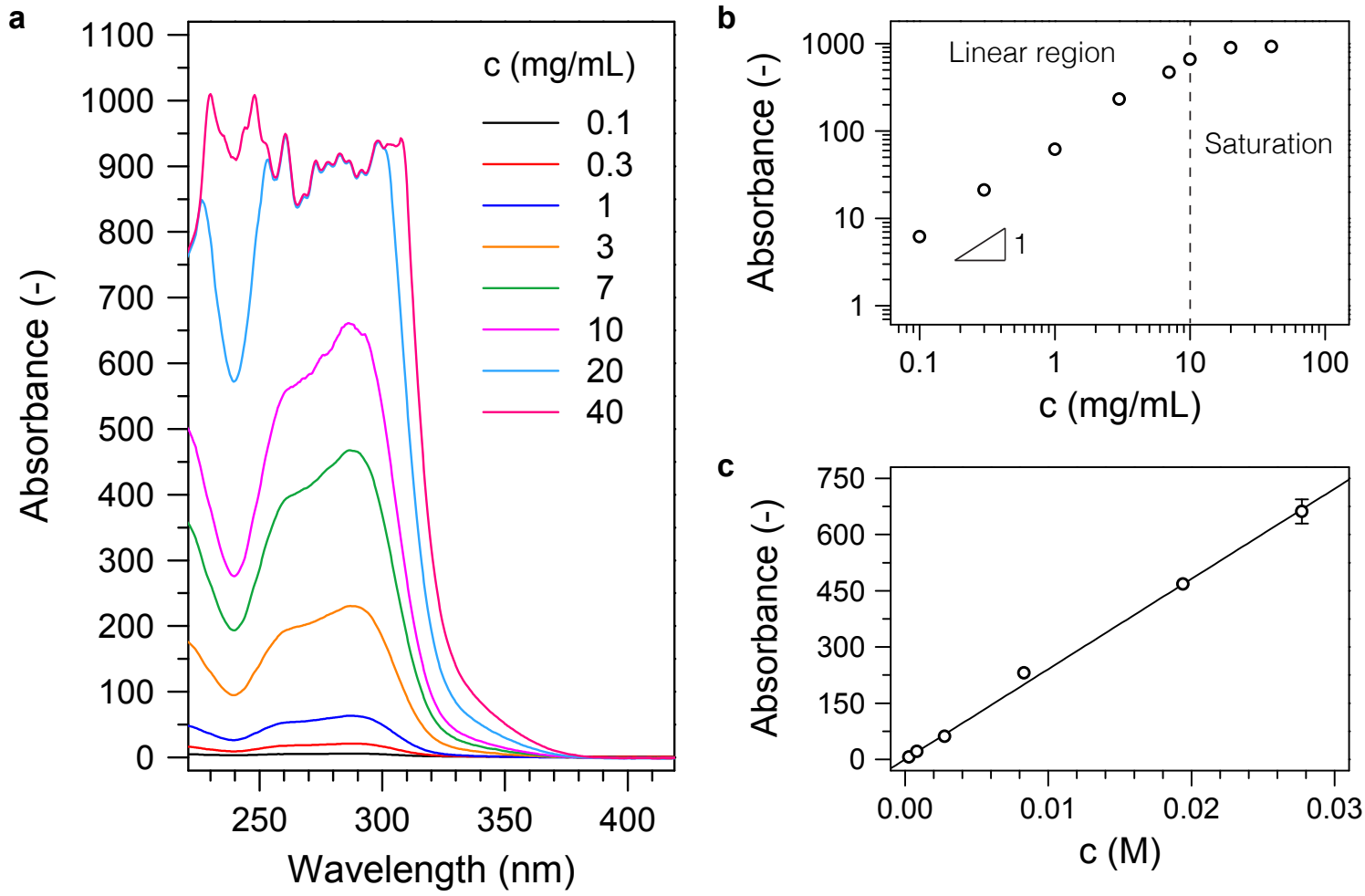

Figure S3. a UV-Vis spectra of fenofibrate in methanol solutions at different concentrations. b Absorbance-concentration plot at $\lambda=287 \mathrm{~nm}$ showing the transition from linear to saturation regions. c Fitting of the data using the Beer-Lambert law in the linear region (concentration is expressed in molar unit).

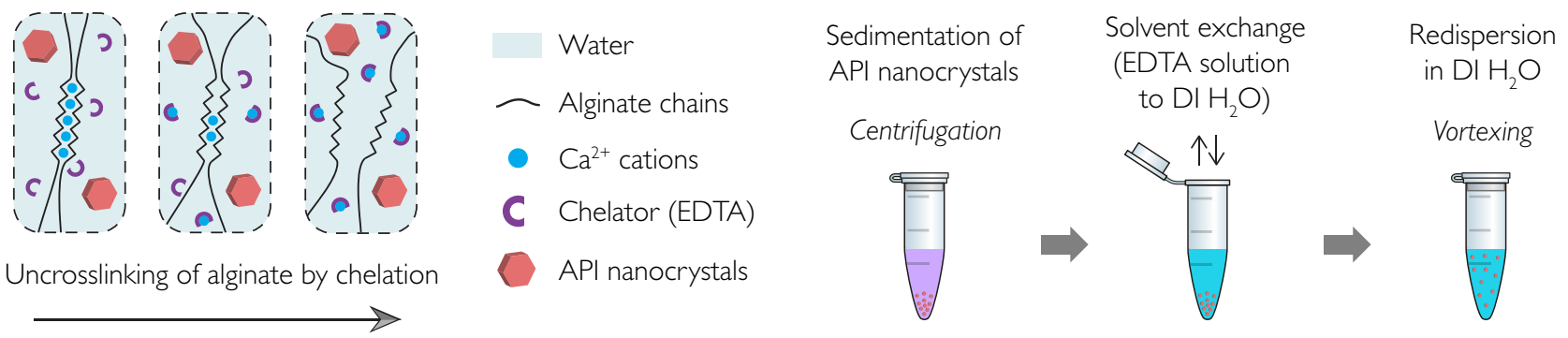

Figure S4. Scheme for the de-crosslinking of the alginate matrix and nanocrystals recovery for TEM observation. 
a

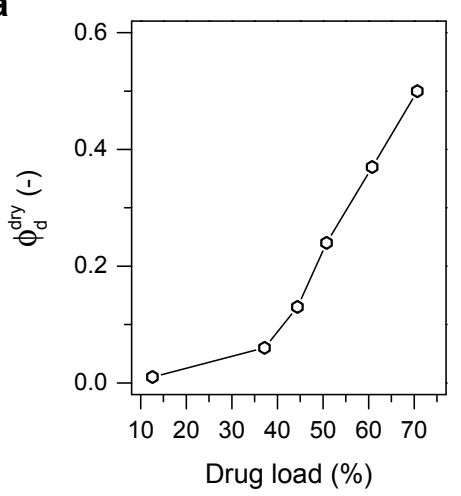

b

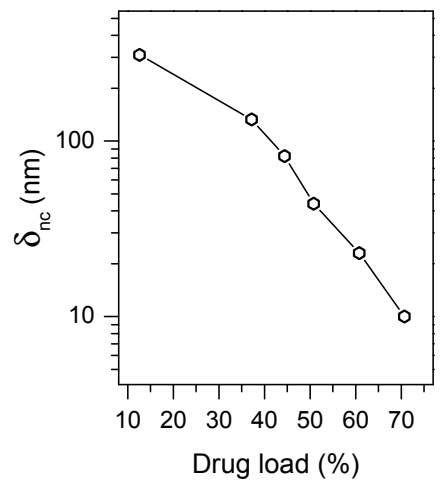

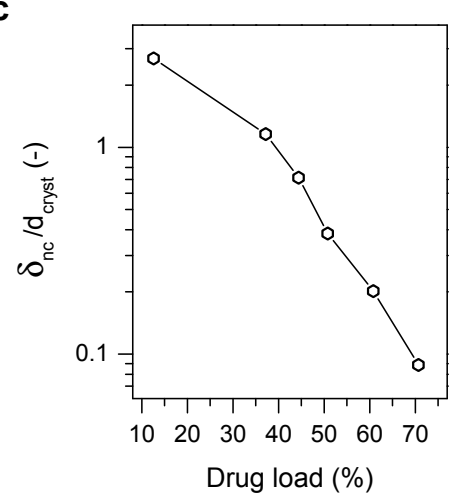

Figure S5. a Volume fraction of the fenofibrate nanocrystals in the dried microparticles, b inter-nanocrystal separation, and $\mathbf{c}$ inter-nanocrystal separation/crystal size ratio as a function of particle drug load.

a

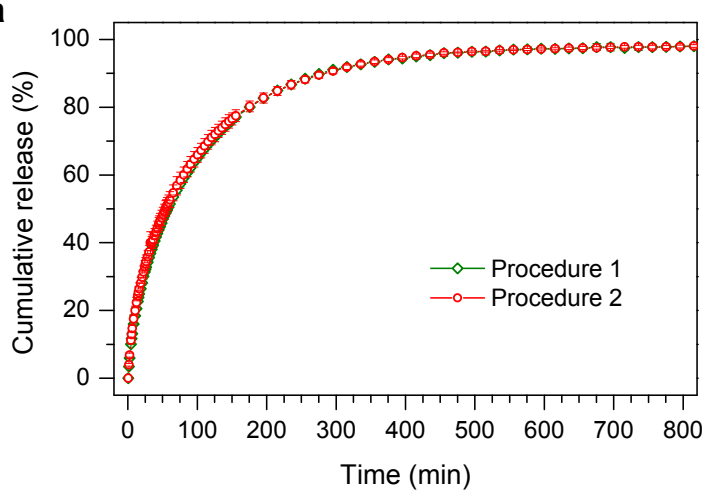

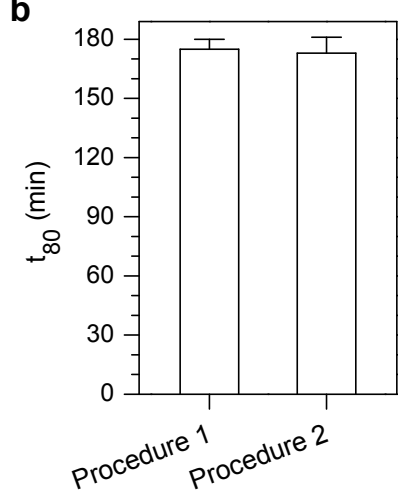

Figure S6. a Cumulative release and $\mathbf{b}$ time at $80 \%$ release for particles at $70.7 \%$ drug load prepared by different procedures. Procedure 1 corresponds to the sieving of dried particles through a $250 \mu \mathrm{m}$ mesh size in order to de-agglomerate the particles. For procedure 2, particles were dried far apart from each other in order to avoid particles agglomeration. The average diameter of the dried particles is $239 \mu \mathrm{m}$ in both cases. 

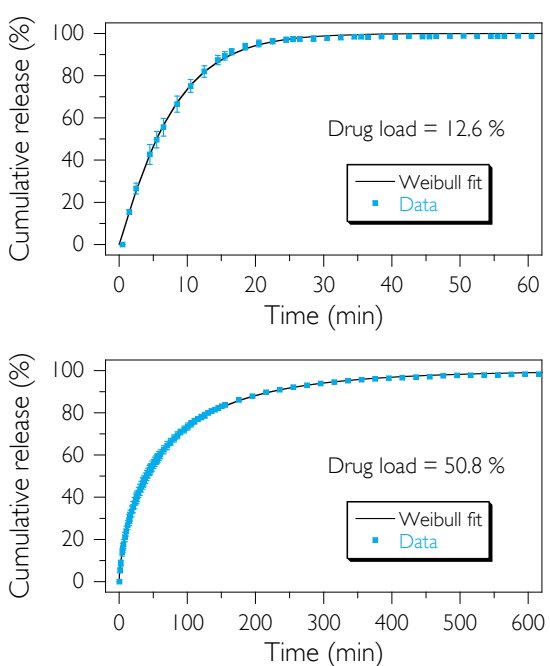

b

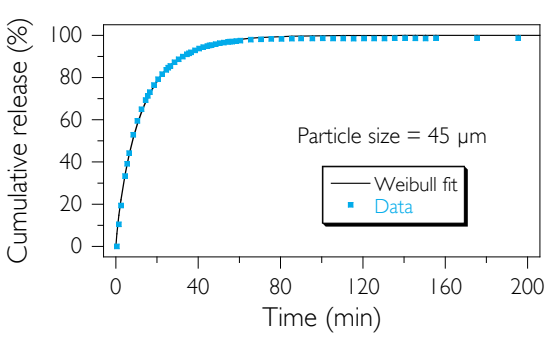

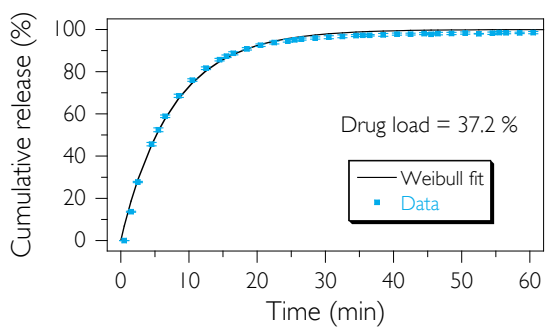
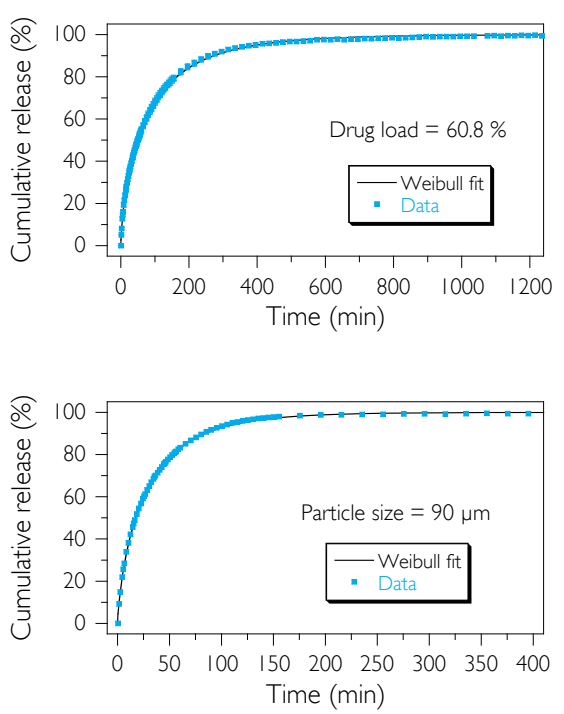
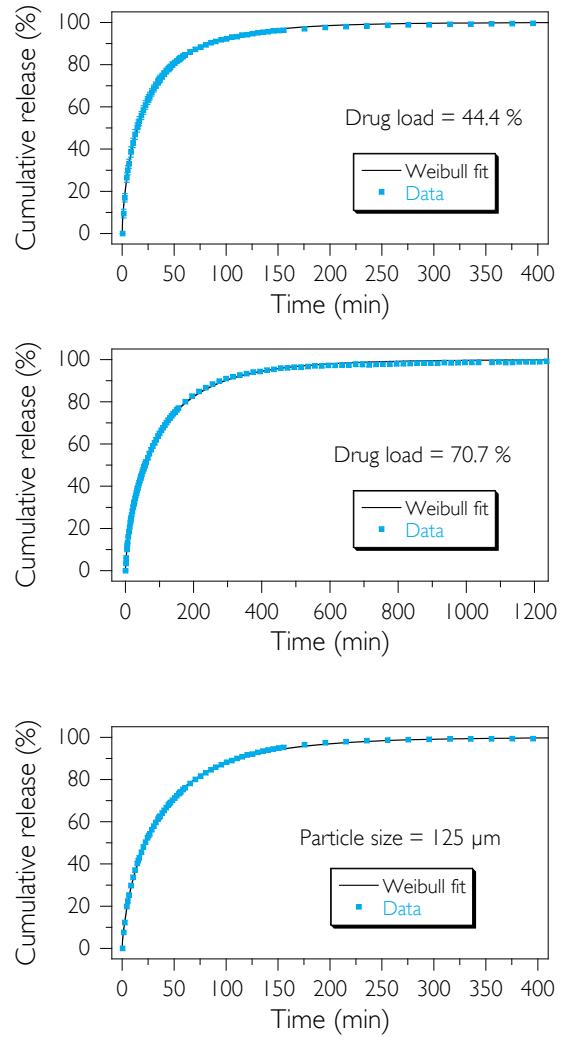

Figure S7. Individual curve fitting of dissolution data of the particles. a Influence of drug load at fixed particle size of $239 \mu \mathrm{m}$ and $\mathbf{b}$ influence of particle size at fixed drug load of $70.7 \%$.

a

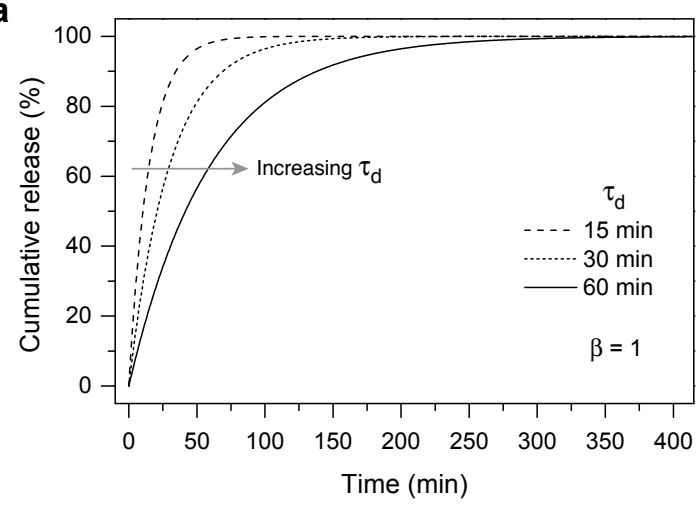

b

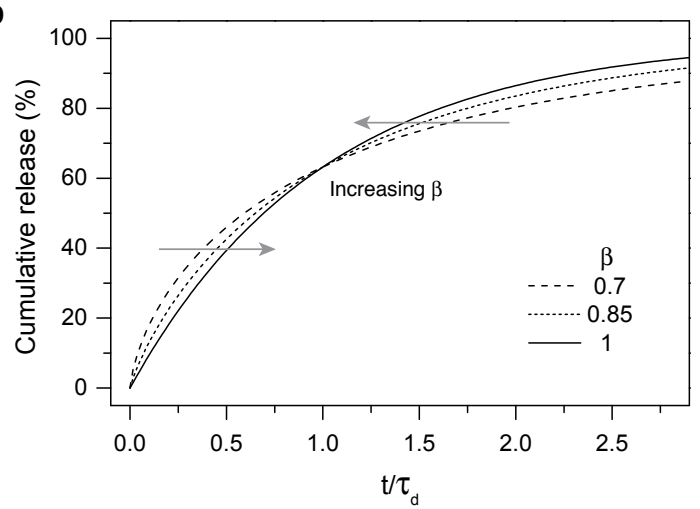

Figure S8. Influence of the Weibull equation parameters on the cumulative release curves. a Influence of the characteristic dissolution time and $\mathbf{b}$ influence of the shape parameter. 
a

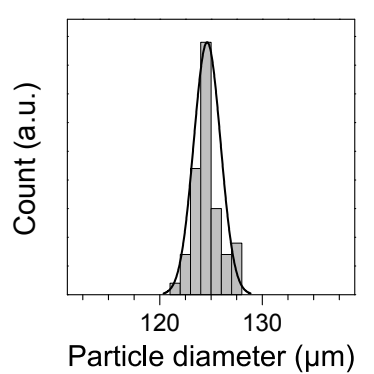

b

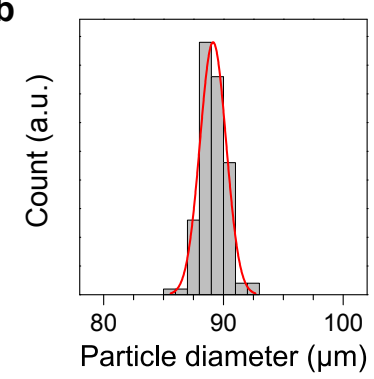

C

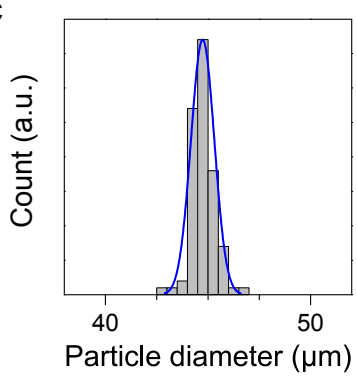

Figure S9. Dried microgel particle size distributions after size reduction of $239 \mu \mathrm{m}$ particles at $70.7 \%$ drug load using sieves with a $125 \mu \mathrm{m}, \mathbf{b} 90 \mu \mathrm{m}$ and c $45 \mu \mathrm{m}$ final mesh size.

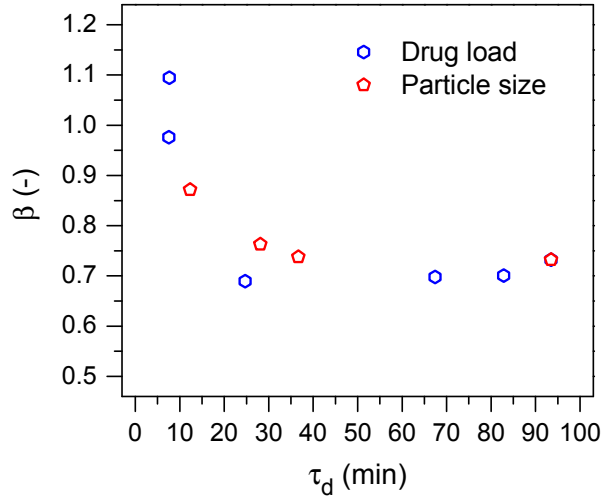

Figure S10. Correlation between the shape parameter $\beta$ and the characteristic dissolution time $\tau_{d}$ of the Weibull function from the fitting of dissolution curves from main Figures 5 and 7 . Blue symbols: variation in drug load; red symbols: variation in particle size. 